\title{
Stricture Rate after Laparoscopic Roux-en-Y Gastric Bypass with a 21-mm Circular Stapler: The Cleveland Clinic Experience
}

\author{
Fahad Alasfar $^{\mathrm{a}}$ Adheesh A. Sabnis $^{\mathrm{b}}$ Rockson C. Liu ${ }^{\mathrm{c}}$ Bipan Chand ${ }^{\mathrm{d}}$ \\ ${ }^{a}$ Department of Surgery, Kuwait University, Kuwait; ${ }^{b}$ Department of Surgery, George Washington University, \\ Washington, D.C., ' Kaiser Permanente, Walnut Creek, Calif., ${ }^{\mathrm{d}}$ The Cleveland Clinic Foundation, Cleveland, Ohio, USA
}

Key Words

Gastric bypass $\cdot$ Stricture $\cdot$ Gastrojejunostomy

\begin{abstract}
Objective: The objectives of this study were to report the incidence of gastrojejunal anastomic strictures that occurred in laparoscopic Roux-en-Y gastric bypass (LRYGB) surgery and to determine the time course of presentation, associated perioperative factors, and response to balloon dilation. Subjects and Methods: All 126 patients who underwent LRYGB at the Cleveland Clinic Foundation between July 2003 and February 2005 were included. We utilized a transoral 21-mm circular stapler for the gastrojejunostomy. Patients with symptoms of anastomotic strictures underwent upper endoscopy by one surgeon (B.C.). A stricture was defined by the inability to pass a $10-\mathrm{mm}$ gastroscope through the anastomosis. Balloon dilation was performed to $12 \mathrm{~mm}$. Records were analyzed retrospectively and statistical analysis including Pearson $\chi^{2}$ statistics, Fisher's exact test and Student's t test were used when appropriate. Results: Symptomatic anastomotic strictures occurred in 29 (23\%) patients. All patients presented with nausea, vomiting and dysphagia. The median time to diagnosis was 52 days (25-309 days). Symptoms resolved after one dilation in $25(86 \%)$ of patients. Two and three dilations were required in 1 (3.5\%) and 3 (10.5\%) of patients, respectively. No patients had complications or required more than 3 dilations. Age, preoperative body mass
\end{abstract}

index (BMI), and intraoperative blood loss did not correlate with stricture formation. Although nonsteroidal anti-inflammatory drugs were used by $46(41 \%)$ of patients after surgery, there was no correlation with stricture formation. Conclusion: Symptomatic anastomotic strictures developed in nearly a quarter of patients who underwent LRYGB utilizing a transoral 21-mm circular stapled gastrojejunal anastomosis. A single endoscopic balloon dilation was usually adequate. Strictures were not predicted by perioperative factors.

Copyright $\odot 2009$ S. Karger AG, Basel

\section{Introduction}

Obesity, as defined by a body mass index (BMI) of more than $30 \mathrm{~kg} / \mathrm{m}^{2}$, is estimated to affect more than 1.5 billion adults [1]. This obesity epidemic has led to a significant increase in the number of bariatric surgeries performed [2]. Since Wittgrove et al. [3] reported 5 cases, laparoscopic Roux-en-Y gastric bypass (LRYGB), has become one of the preferred weight loss procedures. Despite its success, LRYGB continues to be plagued by strictures at the gastrojejunostomy anastomosis that lead to postoperative complications such as vomiting, dysphagia, and patient discomfort. The stricture rate appears to be correlated with the size of the circular stapler [4]. However, the exact incidence of and risk factors leading to

\section{KARGER}

Fax +41613061234 E-Mail karger@karger.ch www.karger.com
(C) 2009 S. Karger AG, Basel

$1011-7571 / 09 / 0185-0364 \$ 26.00 / 0$

Accessible online at:

www.karger.com/mpp
Fahad Alasfar, Assistant Prof.

Department of Surgery, Faculty of Medicine, Kuwait University, PO Box 24923

13110 Safat (Kuwait)

Tel. +965 5319475 , Fax +965 5319597

E-Mail falasfar@hsc.edu.kw or asfar@hotmail.com 
1

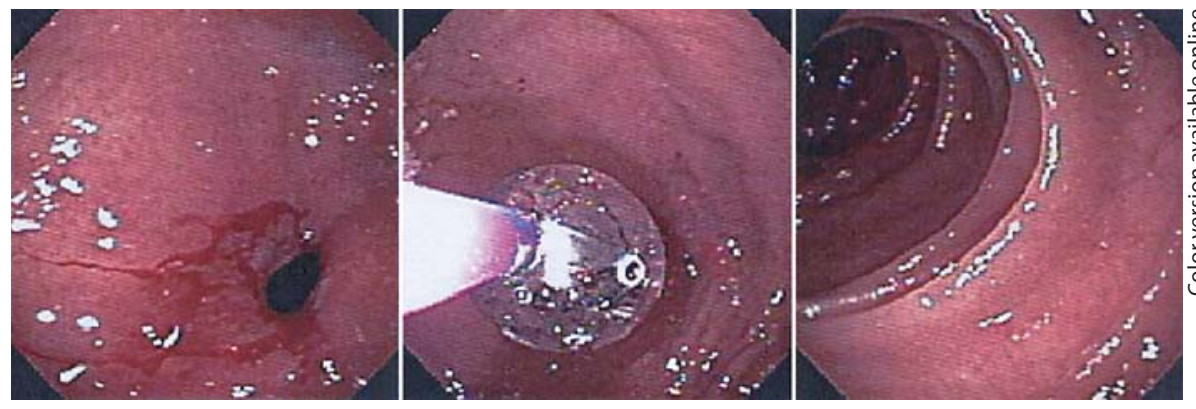

Fig. 1. Gastrojejunostomy balloon dilation and the passage of the endoscope to the jejunum.

Fig. 2. The gastrojejunostomy after dilation.
2

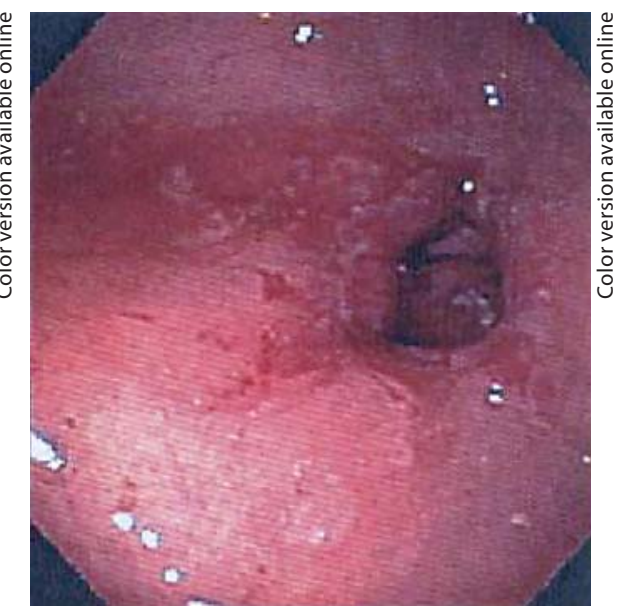

anastomotic strictures remains unclear. In this study, we report the stricture rate after LRYGB performed with a transoral 21-mm circular stapler at the Cleveland Clinic Foundation, and attempt to identify perioperative risk factors.

\section{Subjects and Methods}

A retrospective chart review was undertaken for all patients who had undergone LRYGB at the Cleveland Clinic Foundation in Cleveland, Ohio, USA between July 2003 and February 2005. All operations were performed by 1 surgeon (B.C.). No patients were excluded from this study. All patients met the criteria proposed by the NIH Consensus Panel in 1991 for weight reduction surgery [5].

During the study period, all LRYGBs were performed in a similar fashion by a single surgeon (B.C.). A $30-\mathrm{ml}$ divided gastric pouch was created with several firings of the linear stapler. The biliopancreatic limb was divided $30 \mathrm{~cm}$ from Treitz' ligament. The omentum was split down the midline and wrapped around the $150-\mathrm{cm}$ antecolic, antegastric Roux limb. The gastrojejunostomy was created with a 21-mm ILS (Ethicon Endosurgery, Cincinnati, Ohio, USA) circular stapler with the anvil inserted transorally. The circular stapler was inserted through the LUQ port site which was originally a $12-\mathrm{mm}$ port but later expanded to accommodate the stapler. The gastrojejunostomy anastomosis was not routinely oversewn. Intraoperative upper endoscopy with insufflation was utilized to check the anastomosis for leaks, hemostasis and patency. The mesenteric defect between the jejunojejunostomy was closed with interrupted sutures. Peterson's space was not closed. A routine liver biopsy was performed. A single 10 -french drain is placed in the left upper quadrant. All patients were studied $24 \mathrm{~h}$ postoperatively with routine contrast upper gastrointestinal series (UGI) to evaluate for the presence of anastomotic leak.

Routine upper endoscopy or contrast UGI were not performed following discharge. An anastomotic stricture was suspected if the patient had frequent nausea, emesis and/or dysphagia with liquids or meals. Upper endoscopies were performed liberally in patients with suspected strictures in an outpatient setting with intravenous sedation by 1 of 2 surgeons. Endoscopy was performed using a GIF-160 (10-mm) gastroscope (Olympus America, Inc., Melville, N.Y., USA). A stricture was confirmed by the inability to pass the gastroscope through the gastrojejunal anastomosis. For this study, the stricture size was determined by the endoscopist's dictated note. Dilation is performed if the stricture is less than $10 \mathrm{~mm}$ using a 10- to 12-mm CRE balloon (Cook CRE, Winston-Salem, N.C., USA) for 1 min under sedation without fluoroscopy (fig. 1, 2). Contrast studies were used selectively if patients showed any sign of possible perforation. The gastroscope was passed through the gastrojejunal anastomosis in all patients after dilation.

Variables measured for this study included the incidence of stricture requiring endoscopic dilation, number of dilations required to resolve symptoms, time to diagnosis of stricture, demographic information, estimated blood loss, BMI, American Society of Anesthesiology (ASA) classification, and postoperative use of nonsteroidal anti-inflammatory (NSAID) drugs.

Values for continuous variables are expressed as means and ranges. Univariate analysis was conducted using the Pearson $\chi^{2}$ statistic or Fisher's exact test for categorical data, and the Student's $t$ test for continuous parametric data. Data collection was performed on Microsoft Excel 2003 (Microsoft, Redmond, Wash., USA) and statistical analysis was performed using SPSS 12 (SPSS Inc., Chicago, Ill., USA).

\section{Results}

In this study, 103 females and 23 males with a mean age of 43 years (range 19-61 years) underwent successful elective LRYGB. The patients had an average BMI of 48 $\mathrm{kg} / \mathrm{m}^{2}$ (range $35-75 \mathrm{~kg} / \mathrm{m}^{2}$ ). This series of patients also had significant comorbidities with an average ASA classification of 3 (range 2-4). The mean estimated blood loss 
Table 1. Patient characteristics and perioperative factors

\begin{tabular}{llll}
\hline Characteristics & $\begin{array}{l}\text { Stricture } \\
(\mathrm{n}=29)\end{array}$ & $\begin{array}{l}\text { No stricture } \\
(\mathrm{n}=97)\end{array}$ & Significance \\
\hline Age, years & $44 \pm 9$ & $44 \pm 10$ & NS ( $p>0.93)$ \\
BMI, kg/m $\mathrm{m}^{2}$ & $48 \pm 7$ & $48 \pm 7$ & NS ( $>0.57)$ \\
Estimated blood loss, $\mathrm{ml}$ & $73 \pm 36$ & $83 \pm 54$ & NS ( $>0.33)$ \\
NSAID use & 12 & 34 & NS ( $p>0.72)$ \\
\hline
\end{tabular}

Patient characteristics and perioperative factors were not predictive of anastomotic stricture formation.

was $81 \mathrm{ml}$ (range 25-300 ml) and operative time was 177 min (range 104-347 min). The patients remained in hospital for an average of 3 days (range $2-13$ days). None had leaks on the 24-hour postoperative contrast upper gastrointestinal series.

Of the 126 patients reviewed, 29 (23\%) developed an anastomotic stricture requiring at least 1 dilation (table 1). Of the 29 patients, symptoms resolved after one dilation in 25 (86\%), two dilations in 1 (3.5\%) and three dilations in $3(10.5 \%)$ patients. No patients in our series required more than 3 dilations or operative revision of the anastomosis for their strictures. The median time interval to diagnosis of stricture was 52 days (range 25309). At last follow-up, all patients had complete resolution of symptoms after one, two, or three dilations.

Of the 29 patients who developed a stricture, 12 (41\%) reported NSAID use, $3(10 \%)$ had wound infections requiring incision and draining, 1 (3.5\%) developed a marginal ulcer, and another $1(3.5 \%)$ had a bleed noted at the gastrojejunal anastomosis.

There were no significant $(\mathrm{p}<0.05)$ differences in patients who developed a stricture compared to those who did not with respect to estimated blood loss, preoperative BMI, NSAID use, or age (table 1).

\section{Discussion}

The gastrojejunostomy anastomosis in LRYGB can be performed by three techniques: circular stapled, linear stapled or hand-sewn [6]. Circular stapled anastomosis has been associated with a high stricture rate of $27 \%$ [7] similar to $23 \%$ of our study.

The median time interval between the primary operation and presentation of a stricture was 52 days, which is consistent with the previously published reports $[4,8]$. In our study, strictures developed as late as 10 months postoperatively, similar to data reported by Barba et al. [9]. Many etiologies have been postulated for stricture formation. Ischemia and inflammation are the main factors identified by many investigators [4, 8-10]. Barba et al. [9] noted a correlation between stricture formation and female gender and anastomotic leaks. None of our patients developed postoperative leaks. Other factors like Helicobacter pylori and bile salts have been suggested by Kaplan [10] to have a possible role in stricture formation. Pope et al. [11] found that acid production has no role in stricture formation. They noted that higher stricture rates were associated with a higher number of stapler rows. They postulated that tissue injury is the main factor in the pathophysiology of stricture formation [11]. In our patient population there was no significant difference between the stricture and the no-stricture group with respect to estimated blood loss, preoperative BMI, NSAID use or age. None of our patient population received proton pump inhibitor pre- or postoperatively. We currently are studying the role of proton pump inhibitor in reducing the stricture rate.

Nguyen et al. [4] in a nonrandomized study showed a significant drop in stricture rate with the use of a $25-\mathrm{mm}$ circular stapler instead of a $21-\mathrm{mm}$ one (8.8 from $26.8 \%$ ). However, Stahl et al. [12] showed no significant difference in the stricture rate between the two sizes. Currently the primary surgeon (B.C.) of our study is using a 25mm circular stapler.

In our experience patients with persistent nausea, vomiting and dysphagia with eating after LRYGB frequently have strictures. On the other hand, gastrointestinal complaints such as abdominal pain or indigestion are not associated with strictures [13]. Since specific symptoms correlate well with the presence of a stricture, when we suspect an anastomotic stricture we forgo contrast UGI studies, which are rarely helpful and are uncomfortable for patients. Unlike UGI studies, upper endoscopy is both diagnostic and therapeutic. At the Cleveland Clinic, all patients who present with symptoms suggestive of an anastomotic stricture (nausea, vomiting and dysphagia) undergo upper endoscopy. Dilation with a $12-\mathrm{mm}$ balloon is performed if the anastomosis is less than $10 \mathrm{~mm}$ in diameter.

Balloon dilation of stricture at the gastrojejunostomy has been shown to be safe and effective $[14,15]$. In our series, the endoscopy and dilation were completed safely in all patients. All of the dilations were performed by one surgeon (B.C.), who routinely performs advanced therapeutic endoscopies. Studies have demonstrated the supe- 
rior results of surgeon-performed endoscopic dilation after LRYGB [16].

The majority of patients in our series $(86 \%)$ had complete resolution of their symptoms after only one dilation similar to the findings reported by Barba et al. [9] where $67 \%$ of the patients required only one dilation unlike Rossi et al. [17], who found that more than half of their patients required more than one dilation. In our patient population, only 4 patients (13\%) required more than one dilation. It is possible that our success rate is higher with the initial dilation because we were more aggressive at performing early endoscopy. This has either led to overdiagnosis of anastomotic stricture or early treatment of anastomotic strictures before the maturational phase of wound healing. We suspect it is a combination of the two reasons.

\section{Conclusion}

Our results showed that LRYGB with a 21-mm circular stapler was associated with a $23 \%$ stricture rate, and that the majority were definitively treated with a single endoscopic dilation without complications. Anastomotic strictures did not appear to be related to common perioperative risk factors. More studies are needed to identify the stricture etiology in these patients.

\section{References}

1 Deitel M: Overweight and obesity worldwide now estimated to involve 1.7 billion people. Obes Surg 2003;13:329-330.

$\checkmark 2$ Pope GD, Birkmeyer JD, Finlayson SR: National trends in utilization and in-hospital outcomes of bariatric surgery. J Gastrointest Surg 2002;6:855-860; discussion 861.

$\checkmark 3$ Wittgrove AC, Clark GW, Tremblay LJ: Laparoscopic gastric bypass, Roux-en-Y: preliminary report of five cases. Obes Surg 1994;4: 353-357.

4 Nguyen NT, Stevens CM, Wolfe BM: Incidence and outcome of anastomotic stricture after laparoscopic gastric bypass. J Gastrointest Surg 2003;7:997-1003; discussion 1003.

$\checkmark 5$ National Institutes of Health Consensus Development Panel: Gastrointestinal surgery for severe obesity. Ann Intern Med 1991;115: 956-961.

6 Gonzalez R, Lin E, Venkatesh KR, Bowers SP, Smith CD: Gastrojejunostomy during laparoscopic gastric bypass: analysis of 3 techniques. Arch Surg 2003;138:181-184.
Matthews BD, Sing RF, DeLegge MH, Ponsky JL, Heniford BT: Initial results with a stapled gastrojejunostomy for the laparoscopic isolated Roux-en-Y gastric bypass. Am J Surg 2000;179:476-481.

8 Go MR, Muscarella P 2nd, Needleman BJ, Cook CH, Melvin WS: Endoscopic management of stomal stenosis after Roux-en-Y gastric bypass. Surg Endosc 2004;18:56-59.

-9 Barba CA, Butensky MS, Lorenzo M, Newman R: Endoscopic dilation of gastroesophageal anastomosis stricture after gastric bypass. Surg Endosc 2003;17:416-420.

$>10$ Kaplan LM: Gastrointestinal management of the bariatric surgery patient. Gastroenterol Clin North Am 2005;34:105-125.

11 Pope GD, Goodney PP, Burchard KW: Peptic ulcer/stricture after gastric bypass: a comparison of technique and acid suppression variables. Obes Surg 2002;12:30-33.

12 Stahl RD, Sherer RA, Seevers CE, Johnston $\mathrm{D}$ : Comparison of 21 vs. $25 \mathrm{~mm}$ gastrojejunostomy in the gastric bypass procedure early results. Obes Surg 2000;10:540-542.
13 Huang CS, Forse RA, Jacobson BC, Farraye FA: Endoscopic findings and their clinical correlations in patients with symptoms after gastric bypass surgery. Gastrointest Endosc 2003;58:859-866.

14 Ahmad J, Martin J, Ikramuddin S, Schauer P, Slivka A: Endoscopic balloon dilation of gastroenteric anastomotic stricture after laparoscopic gastric bypass. Endoscopy 2003;35:725-728.

15 Sanyal AJ, Sugerman HJ, Kellum JM, Engle KM, Wolfe L: Stomal complications of gastric bypass: incidence and outcome of therapy. Am J Gastroenterol 1992;87:1165-1169.

16 Bell RL, Reinhardt KE, Flowers JL: Surgeonperformed endoscopic dilatation of symptomatic gastrojejunal anastomotic strictures following laparoscopic Roux-en-Y gastric bypass. Obes Surg 2003;13:728-733.

-17 Rossi TR, Dynda DI, Estes NC, Marshall JS: Stricture dilation after laparoscopic RouxenY gastric bypass. Am J Surg 2005;189:357360 . 